\title{
A INCLUSÃO DA INTERNET NA EDUCAÇÃO PÚBLICA
}

\author{
THE INCLUSION OF THE INTERNET IN PUBLIC EDUCATION
}

\author{
Abilene San Martin de Souza ${ }^{1}$ \\ Cleonildo de Lima Campos ${ }^{2}$ \\ Juniana Patrícia Silva de Souza ${ }^{3}$ \\ Maria Auxiliadora da Silva Faria Garcia ${ }^{4}$ \\ Rosecler de Carvalho Senra Santos ${ }^{5}$ \\ Silvaneide da Silva Vieira ${ }^{6}$
}

RESUMO: O objetivo do presente artigo é, abordar a importância da internet na educação, foi utilizado a metodologia bibliográfica realizada por meio de revistas como a veja, livro como (metodologias ativas para uma educação inovadora: uma abordagem teórico-prática como e alguns sites para desenvolvimento e complementação do trabalho como Educação no século XXI: Cognição, tecnologias e aprendizagens. Atualmente, sabemos bem que o uso das tecnologias no ensino precisa vencer inúmeras barreiras, como a falta de infraestrutura, a falta de máquinas (computadores) nas escolas, internet de qualidade e com uma boa velocidade além de investimentos em capacitação para os funcionários. Aliás, a capacitação de funcionários é um dos desafios que permeiam e impedem a evolução da internet na educação pública em nosso país. Atualmente, várias escolas no Brasil foram beneficiadas com um sistema de informação avançado a internet, onde o MEC desenvolveu diversos projetos como o Programa Nacional de Tecnologia Educacional (Proinfo) que disponibiliza computadores, recursos digitais e conteúdos educacionais às escolas além do projeto Um Computador por Aluno (UCA), que contribui com notebooks para todos os estudantes do ensino médio, em especial aos alunos Portadores de Necessidades Especiais (PNE). Para promover o acesso à internet há ainda o Programa de Banda Larga nas Escolas (PBLE) e outras ações, como o Programa Nacional de Formação Continuada presentes em todas as

\footnotetext{
I Licenciatura em Pedagogia pela "Feics", (Faculdades Evangélicas Integradas Cantares de Salomão). Especialização "Educacao infantil com ênfase em Educação Especial. AVEC Faculdades Integradas de Várzea Grande.

2 Bacharel em Direito pela UNIC ( Universidade de Cuiabá),, licenciado em Pedagogia pelo Centro Universitário Uninter, Pós Graduado em Educação Infantil e Psicopedagogia Pela Faculeste.

${ }^{3}$ Licenciada em pedagogia pela UNIVAG ( Universidade de Várzea Grande).

${ }^{4}$ Licenciada em Pedagogia.

${ }^{5}$ Faculdade de licenciatura plena em pedagogia realizada na universidade federal UFMT. especialização em educação infantil realizada na UFMT especialização em psicopedagogia realizada na FAUC.

6 Licenciatura em Pedagogia pela "Feics", (Faculdades Evangélicas Integradas Cantares de Salomão). Especialização "Educacao infantil com ênfase em Educação Especial. AVEC Faculdades Integradas de Várzea Grande.
} 
instituições municipais e estaduais, a Tecnologia Educacional (Proinfo Integrado) que orientam os educadores sobre o uso correto dessa tecnologia inovadora com seus alunos em sala de aula e todo o ambiente escolar. Em contra partida o MEC está atento para as necessidades das escolas estarem conectadas e de tecnologias pedagógicas serem disseminadas na rede pública de ensino. A direção é esta, mas ainda falta muito aprimoramento para o uso correto dessa ferramenta inovadora a Internet. Nota-se que a necessidade de evolução está presente em todo o mundo hoje, inclusive nos meios educacionais, a qual boa parte das instituições de ensino e aprendizagem conta com softwares e sites web para os desenvolvimentos de atividades complementares e vídeos aulas competentes que contribuirão na formação e na orientação de seus alunos.

Palavras-chave: MEC. Internet. Computadores.

ABSTRACT: The aim of this article is to address the importance of the internet in education, using the bibliographic methodology carried out on top of magazine magazines and some sites for the development and complementation of the work. Nowadays, we are well aware that the use of technologies in education needs to overcome numerous barriers, among them the issue of infrastructure, the lack of machines (computers) in schools, quality internet and with a good speed in addition to investments in training for employees. In fact, the training of employees is one of the challenges that permeate and hinder the evolution of the internet in public education in our country. Currently, several schools in Brazil have benefited from an advanced information system on the internet, where MEC has developed several projects such as the National Educational Technology Program (Proinfo) that makes computers, digital resources and educational content available to schools in addition to the project Um Computer por Student (UCA), who contributes notebooks for all high school students, especially students with Special Needs (PNE). To promote access to the Internet, there is also the Broadband in Schools Program (PBLE) and other actions, such as the National Continuing Education Program present in all municipal and state institutions, Educational Technology (Proinfo Integrado) that guide educators on the correct use of this innovative technology with its students in the classroom and the entire school environment. In contrast, MEC is aware of the need for schools to be connected and for pedagogical technologies to be disseminated in the public school system. This is the direction, but there is still much improvement for the correct use of this innovative tool on the Internet. It is noted that the need for evolution is present all over the world today, including in educational environments, which a large part of teaching and learning institutions have software and websites for the development of complementary activities and competent video lessons that will contribute to the training and orientation of its students.

Keywords: MEC. Internet. Computers. 


\section{INTRODUÇÃO}

Atualmente a educação pública do Brasil vem passando por uma grande transformação, onde meios de informação e comunicação tem sido uma das ferramentas de grande inovação dentro das salas de aulas das escolas, seja ela a estadual ou municipal.

O objetivo desse artigo é abordar a importância da internet na educação, seja ela pública ou particular desde as séries inicias até o ensino médio. Atualmente, sabemos bem que o uso das tecnologias no ensino precisa vencer inúmeras barreiras, entre elas a questão de infraestrutura, a falta de máquinas (computadores) nas escolas, internet de qualidade e com uma boa velocidade além de investimentos em capacitação para os funcionários A internet tem aberto portas para a motivação do aprendizado em sala de aula, os meios de ensino abordados por diversos professores tem sido a inovação, proporcionando aos alunos aulas interativas com tele vídeos e atividades online.

Nota-se que a internet vem quebrando uma grande barreira de superação de inovação e tecnologia na educação pública.

Podemos dizer que a internet, será a ferramenta complementar mais ágil nos ambientes escolares se implantada de forma correta, facilitando o diálogo, o intercâmbio de informações entre pessoas de estados e países diferentes.

De acordo com a pesquisa realizada em alguns livros, sites e revistas como proinfo, educa+, para a adaptação da internet na sala de aula será necessária grande atenção com os alunos como o acesso seguro a sites e aplicativos educacionais, pois sabemos que a segurança será mais que um requisito necessário, a falta de profissionais qualificados é uma das características que permeiam essa grande transformação nos ambientes escolares hoje.

\section{REFERENCIAL TEÓRICO}

Cogita-se, com muita frequência, a questão da internet na educação, uma ferramenta que a cada dia que passa vem crescendo diante de uma sociedade consumidora de informações, muitas delas virtuais. 


\begin{abstract}
Obviamente, dotar as escolas de computadores, melhorar o acesso à internet e capacitar professores e alunos para o uso da informática são ações importantes para promover a inclusão digital e democratizar o acesso a informações indispensáveis para entender o mundo que nos cerca. Resta saber como o uso dos computadores poderá de fato fazer diferença na aprendizagem. (CASTRO, 2oro, p. 65).
\end{abstract}

As redes de ensino estão cada vez mais tentanto se adequar a grande ideia de implantar a internet nas salas de aulas das escolas públicas do Brasil, uma ação que se bem desenvolvida irá beneficiar toda a comunidade escolar.

[...] a minha questão não é acabar com a escola, é mudá-la completamente, é radicalmente fazer que nasça dela um novo ser tão atual quanto à tecnologia. Eu continuo lutando no sentido de pôr a escola à altura do seu tempo. E pôr a escola à altura do seu tempo não é soterra-la, mas refazê-la (FREIRE \& PAPERT, 1996, p. 245).

A internet tem sido hoje uma das ferramentas mais promissoras, causando transformações tanto os ambientes profissionais (trabalho) quanto em muitas outras organizações e instituições, como faculdades e escolas públicas do nosso país.

\title{
2.I Os problemas encontrados para a implantação da internet em muitas das unidades escolares
}

Alguns dos grandes problemas encontrados estão relacionados à manutenção dos computadores, o valor monetário necessário para mantê-los funcionando dentro das instituições, técnicos qualificados e uma estrutura adequada as necessidades e que ampare toda a demanda de aluno. Frisamos aqui também o monitoramento necessário dos programas, as licenças de muitos componentes e um dos principais pontos a privação da rede para acesso a outros sites que não estão disponibilizados para o uso no ambiente tais como sites de relacionamento, bate-papo, redes sociais etc.

\footnotetext{
A educação não se reduz à técnica, mas não se faz educação sem ela. Utilizar computadores na educação, em lugar de reduzir, pode expandir a capacidade crítica e criativa de nossos meninos e meninas. Dependendo de quem o usa, a favor de que e de quem e para quê. O homem concreto deve se instrumentar com o recurso da ciência e da tecnologia para melhor lutar pela causa de sua humanização e de sua libertação. (FREIRE, 2001a, p.98).
}

Outro fator a ser analisado em muitas instituições de ensino é a questão da internet, muitas vezes de baixa velocidade e pouquíssima qualidade que inviabilizam a navegação na web, privando o acesso a vídeos aulas disponibilizado pelas escolas entre 
outras competências e atividades que podem contribuir além de agregar maior conhecimento aos alunos.

Para ficar mais claro, citamos aqui alguns requisitos necessários para a implantação hoje da internet nas escolas públicas, requisitos básico que merecem uma importante atenção.

- Manutenção dos equipamentos: A responsabilidade dos órgãos públicos na manutenção dos equipamentos das escolas, é mais que necessário, é uma das questões mais importantes, e uma ação que muitas vezes contribui para essa ferramenta não evoluir dentro dos ambientes públicos.

- Programas, e aulas para a formação dos docentes: os programas de formação em TICs para professores dos sistemas de ensino, é mais que obrigatório, além de proporcionar a oportunidade de aprofundar o conhecimento nas áreas os professores poderão ter mais uma nova formação em seus currículos;

- Disponibilização de conteúdos educativos: Os conteúdos que serão disponibilizados para os alunos são de total responsabilidade dos professores, isso tudo passará a fazer parte dos seus planos e aulas efetivamente, onde é de suma importância a atenção e a segurança de seus alunos.

Alguns indicadores que fazem parte da escola, e que passará a ser requisitos necessários têm-se as seguintes variáveis:

- O Perfil da escola

- Perfil do entrevistado

- Infraestrutura disponível

- Manutenção técnica

- Perfil de usuário

- Casos de uso 
Quanto aos indicadores que norteiam o eixo do instrutor cabe aqui toda a infraestrutura escolar onde os computadores estarão disponíveis para uso dos professores, para desenvolvimento de seus planos de aulas, e a aplicação aos seus alunos.

- Disponibilidade

- Fluência digital

- Modelo de uso

- Relação uso do computador e processo de construção do conhecimento.

\section{I.I Educação para o futuro}

A internet tem se tornado a principal ferramenta nos ambientes corporativos e de ensino, fazendo com que a educação brasileira desse um grande salto em desenvolvimento dos alunos e métodos de aprendizagem abordados pelos professores dentro e fora de sala de aula.

De acordo com Algumas instituições de ensino tais como; o Colégio Isac Newton, Faculdade Anhanguera Educacional entre outras, já conta com uma base virtual ou ambiente virtual de aprendizagem conhecido como (AVA) para disponibilização de conteúdos acadêmicos complementares, nos portais eletrônicos e sites institucionais, além de materiais e avaliações de aprendizagem. São algumas ferramentas que as instituições de ensino já se adequaram para fazer uma educação mais evoluída.

Percebe-se que boa parte das instituições de ensino seja ela municipal ou estadual contam hoje com vários outros meios além dos livros didáticos, como blogs, e sites para auxiliar nas atividades propostas pelos professores.

\section{METODOLOGIA DE PESQUISA}

A metodologia abordada foi um levantamento elaborado com base em livros como como o "Poder da Internet: O Encontro de Dois Mundos", escrito por Tiago Baeta e Nathalia Torezani, revistas como A Veja, e pesquisas na internet como apontam as referências Bibliográficas, onde foi constatado que, para a implantação de uma educação de 
qualidade e prática nas instituições de ensino e aprendizagem com uso da tecnologia da informação em sala de aulas será necessário as escolas passar por uma grande reforma, incluindo as estruturas (prediais) e formação específica para alguns membros do pessoal.

Neste sentido, Bonilla afirma que:

As TIC, mais do que um simples avanço no desenvolvimento da técnica, representam uma virada conceitual, à medida que essas tecnologias não são mais apenas uma extensão dos sentidos humanos, onde o logos do fazer, um fazer mais e melhor, compõe a visão do mundo. As tecnologias da informação e comunicação são tecnologias intelectuais, pois ao operarem com proposições passam a operar sobre o próprio pensamento, um pensamento que é coletivo, que se encontra disperso, horizontalmente, na estrutura em rede da sociedade contemporânea. (2005, p. 21).

A pesquisa para realização deste artigo é qualitativa, pois se trata de uma pesquisa etnográfica aplicada a educação, onde primeiramente foi realizado um levantamento bibliográfico que apurou resultados de pesquisas nacionais em alguns sites como, Planeta Educação, Escola Educação, brasil escola, info+educação, e-mec, envolvendo o uso de computadores e internet nas escolas de ensino básico e que permitisse identificar as necessidades além de indicar fatores considerados relevantes no uso de computadores na educação pública. Com isso analisamos alguns pontos como a infraestrutura, o uso dos computadores e o seu uso nas escolas, seja na visão das políticas públicas, quanto da escola e dos professores.

\section{RESULTADOS E DISCUSSÕES}

A preparação de gestores hoje ainda é um grande problema em boa parte das instituições de ensino presente em nosso país, o instrutor, como o principal responsável dentro das salas de aulas, com utilização da internet, será mais que necessário estar ligado à segurança e bem estar dos alunos, sabemos bem que a internet possui a sua máscara e utilizar essa ferramenta como meio de entretenimento, livre navegação, poderá gerar risco para todos aqueles conectados. Sendo assim, é o principal papel do professor evitar que os alunos sejam dispersos enquanto realizam suas pesquisas dentro das salas de aulas. Isso mostra um dilema complicado de ser resolvido na prática, pelo fato de que, quando os alunos descobrem uma coisa nova, sempre há uns que são dispersos com as atividades 
proposta, sendo assim é mais que obrigatório à vedação de muitos sites que não irão contribuir para esse processo de transformação na educação pública, como os sites de relacionamentos, bate papos, e sites impróprios para o ambiente.

Conforme Oliveira (1997); "Alunos e professores precisam receber incentivo a fim de que possam utilizar as novas tecnologias, com objetivo de contemplar as necessidades educacionais". (OLIVEIRA,I997, p. 34)

Como citam Holbig et al. (2006);“É uma ferramenta para pesquisa e comunicação, aprendizado e troca de ideias”. (HOLBING, 2006, p. 28).

A Internet está abrindo as portas cada dia mais para que a educação passe a ter uma extensão no meio virtual, para isso estão surgindo cada vez mais novos sites interativos, vários programas que possibilitam o acesso dos alunos no mundo virtual de forma mais ampla e inovadora, muitas escolas, colégios hoje já possuem portais virtuais onde os alunos possam estar realizando atividades e aprimorando mais os conhecimentos partir de web aulas disponibilizadas.

Além disso, poderá ajudar o professor no preparo de suas aulas, tendo este acesso aos artigos atualizados, às notícias mais recentes, pode pedir ajuda aos colegas conhecidos ou desconhecidos e o professor se torna um assessor próximo do aluno, mesmo quando não está fisicamente presente, permitindo coordenar o processo de ensino aprendizagem, estimulando e acompanhando a pesquisa, debatendo os resultados.

A internet facilita a pesquisa aos alunos e o preparo da apresentação dos trabalhos tanto individual como em grupo, possibilitando a consulta a colegas conhecidos ou não de sua região ou de outras partes do mundo. Sabemos bem que essa ferramenta, a Internet, irá incentivar e facilitar muito mais além, a interação dos alunos na troca de informações e resultados, podendo também contribuir para a prática de muitas línguas estrangeiras, e principalmente o aperfeiçoamento em seus conhecimentos. Ela poderá possibilitar a abertura de um ensino e uma aprendizagem dentro dos processos mais abertos, flexíveis, inovadores e contínuos, onde o conhecimento dos alunos será reconhecido e a evolução será a chave do sucesso nos ambientes escolares, principalmente nos ambientes de ensino Público. 


\section{CONCLUSÃO}

De acordo com este artigo desenvolvido podemos afirmar a partir dos levantamentos feitos que, para a implantação da internet na educação pública do País, aconteça é necessário, alguns requisitos básicos mais essenciais como tais como, uma boa internet, programas de desenvolvimento educacional que aborde esse novo ramo, mídias digitais, uma infraestrutura escolar de qualidade com salas amplas, manutenção frequente nos equipamentos eletrônicos, a segurança, a responsabilidade dos profissionais, e o principal de todos, a qualificação necessária dos professores para essa nova fase, mudança, transformação, em fim esse novo ciclo que irá fazer a mudança não somente na vida e no conhecimento dos alunos, mais na capacitação também de todos aqueles que fazem parte da organização.

Durante a elaboração desse trabalho alguns meios sobre o uso das TICs (Tecnologia da Informação e Comunicação) nas escolas públicas do nosso país, como a plataforma de ensino, que as escolas terão que adotar para essa educação do futur, análise dos dados deste trabalho permitiu apontar alguns acertos e alguns problemas comuns no uso de computadores e da internet na educação básica, pública em nosso país, na maioria das escolas, as atividades que utilizam tecnologia e são realizadas com os alunos têm pouca complexidade ou usam ainda recursos simples.

A integração da internet em sala de aula, principalmente na educação pública, tende a evoluir os nossos alunos, assim se de forma correta utilizada, a vontade de aprender mais e mais, além é claro de ficarem informatizados de todos os assuntos em relação à educação, atividades disponibilizadas, concursos e até mesmo debates online, como uma espécie de jogos didáticos. A utilização de equipamentos eletrônicos em aulas facilita a aprendizagem quando de forma correta é utilizada, cito aqui alguns sites com sugestões de vídeos que frisam contribuir de maneira indireta, o YOUTUBE, VEVO, são alguns sites onde não pagam taxa monetária alguma e poderá ser implementado, em muitas escolas que poderiam contar com Web Aulas, fazendo assim uma aula mais produtiva, frisamos novamente, para que isso aconteça é necessário um profissional de qualidade com uma ótima formação. 


\section{REFERÊNCIAS}

FREIRE, "A formação de educadores em serviço com foco nas práticas escolares com o uso do laptop educacional em uma escola pública". In: XIX Simpósio Brasileiro de Informática na Educação, 2008, Fortaleza. Sbie Tecnologia e educação para todos. Fortaleza: Universidade Federal do Ceará, 2008. Disponível em: 〈http://www.fvc.org.br/estudos-epesquisas/avulsas/estudosi-7-uso-computadores $>$ Acesso emi4/o4/202I.

ANDRADE, P. F. \& LIMA, M. C. M. Programa Nacional de Informática Educativa. A utilização da informática na escola pública brasileira (1970-2004). MEC: Secretaria de Educação a distância, 1996 - FREIRE \& PAPERT, 1996.

ARRUDA, R. V.; SILVA, W. A.; LAMOUNIER, E. A.; RIBEIRO, M. W.; CARDOSO, A. \& FORTES, N. "Realidade virtual não-imersiva como tecnologia de apoio no desenvolvimento de protótipos para reconstituição de ambientes históricos para auxílio ao ensino". V Workshop de Realidade Virtual e Aumentada / MATERIAL APOIO WRVA 2008, UNESP-Bauru, 2008.

CASTRO. "Computers and Student Learning: Interpreting the Multivariate Analysis of PISA 2000", Journal of Research on Technology in Education, vol. 37, no 4, 2005.

BONILLA. "Atributos escolares e o desempenho dos estudantes: uma análise em painel dos dados do Saeb". In: Instituto Nacional de Estudos e Pesquisas Educacionais Anísio Teixeira (Inep)/MEC, Brasília, ISSN: I4I4-0640, 2007. BONILLA (2005, p. 21).

BITTENCOURT, J. Atividades desenvolvidas pelo LEC/UFRGS na Escola Luciana de Abreu. Rio de Janeiro: LEC/UFRGS. Slides, color, apresentação multimídia, 2008. 
CASTRO. "Robótica na periferia? Uso de tecnologias digitais na rede pública de ensino de São Paulo como ferramenta de expressão e inclusão". In: XI Workshop de Informática em Educação - WIE, Simpósio Brasileiro de Computação, São Leopoldo-RS, jul. 2005.

CASTRO, M. F. D \& ALVES, L. A. "Avaliação da implementação, uso dos computadores e formação dos professores das escolas públicas de Niterói/RJ". In: III Seminário Internacional: As Redes de Conhecimento e a Tecnologia, UFRJ, 2005.

CASTRO, M. H. G. "A consolidação da política de avaliação da educação básica no Brasil". Revista Meta Avaliação, Rio de Janeiro, v. I, no 3, págs. 271-296, set. Dez. 2009.

ESCOLA PUBLICA. Educação para o futuro. Disponível em: 〈http://revistaescolapublica.uol.com.br/textos/36/educacao-para-o-futuro-302282-I.asp〉 Fortaleza: Universidade Federal do Ceará, 2008.

Levantamento analise sobre o site. Disponível em: <http://objetoseducacionais2.mec.gov.br/> Acesso em: 21/o9/2015.

HOLBING, 2006, p. 268 , J. Atividades desenvolvidas pelo Escola. Rio de Janeiro: LEC/UFRGS. Slides, color, apresentação multimídia, HOLBING, 2006) 